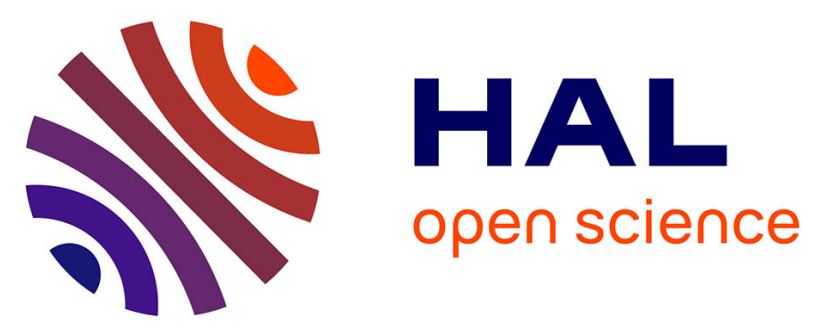

\title{
Modeling of 'quench' or the occurrence and propagation of dissipative zones in REBCO high temperature superconducting coils
}

\author{
Arnaud Badel, Blandine Rozier, Brahim Ramdane, Gérard Meunier, Pascal \\ Tixador
}

\section{To cite this version:}

Arnaud Badel, Blandine Rozier, Brahim Ramdane, Gérard Meunier, Pascal Tixador. Modeling of 'quench' or the occurrence and propagation of dissipative zones in REBCO high temperature superconducting coils. Superconductor Science \& Technology, 2019, 32 (9), pp.094001. 10.1088/13616668/ab181f . hal-02509494

\section{HAL Id: hal-02509494 \\ https://hal.science/hal-02509494}

Submitted on 30 Mar 2021

HAL is a multi-disciplinary open access archive for the deposit and dissemination of scientific research documents, whether they are published or not. The documents may come from teaching and research institutions in France or abroad, or from public or private research centers.
L'archive ouverte pluridisciplinaire HAL, est destinée au dépôt et à la diffusion de documents scientifiques de niveau recherche, publiés ou non, émanant des établissements d'enseignement et de recherche français ou étrangers, des laboratoires publics ou privés. 


\title{
Modeling of 'quench' or the occurrence and propagation of dissipative zones in REBCO high temperature superconducting coils
}

\author{
Arnaud Badel $\left(\mathbb{D}\right.$, Blandine Rozier ${ }^{\circ}$, Brahim Ramdane, \\ Gérard Meunier $(1)$ and Pascal Tixador \\ Univ. Grenoble Alpes, CNRS, Grenoble INP, G2ELab, 38000 Grenoble, France \\ E-mail: arnaud.badel@neel.cnrs.fr
}

\begin{abstract}
Thanks to their very high current carrying capabilities even under high magnetic field conditions and their outstanding mechanical properties, high temperature superconductors (HTSs) such as REBCO (rare-earth $\mathrm{BaCuO}$ ) tapes are very attractive for high magnetic field applications.

Depending on the magnet design goals and constraints, it can be advantageous in some cases to reduce the electrical margins of the conductor. Considering the uncertainty in locally evaluating the critical current, and the inhomogeneities of present-day REBCO tapes, there is a significant risk of the critical current being overstepped locally, thus triggering local damaging hotspots. Such an event does not have the sudden occurrence and/or fast spreading quality usually associated with the concept of 'quench' and should be simply seen as thermal runaway induced by dissipative zones (DZs). The development of numerical models to evaluate the occurrence and propagation of such zones inside windings is critical in the development of a HTS magnet fully using REBCO tape performance while guaranteeing safe operation conditions. In this work, we have developed a transient electro-thermal model adapted to pancake-based coils. It accounts for both the nonlinear electrical and thermal behavior of the material and considers the local inhomogeneities of the critical current $I_{\mathrm{c}}$ along the tape. The electrical part is one-dimensional (1D) and computes the nonlinear dissipation in the conductor depending on the local operation conditions while the thermal part is two-dimensional (2D) to account for the heat propagation along the conductor length and from turn to turn. In order to improve computation efficiency, adaptive time-stepping methods have been introduced with the objective of ensuring good accuracy of simulation results.
\end{abstract}

Keywords: HTS magnets, quench propagation, HTS modeling, REBCO

\section{Introduction}

REBCO (rare-earth $\mathrm{BaCuO}$ ) materials exhibit good mechanical strength and high current carrying capabilities without dissipation even under magnetic fields of $30 \mathrm{~T}$ and beyond [1] when operating at $4.2 \mathrm{~K}$, making them particularly attractive for high magnetic field generation. REBCO-coated conductors tapes are now available in hundreds of meters, and the first large-scale high temperature superconductor (HTS) magnets have started to appear [2-4].
HTS magnets are very stable at any operating temperature. At low temperature (4.2-10 K range), the thermal inertia is small, as in low temperature superconductor (LTS) magnets, but the temperature margin is much bigger, so that the temperature increase to trigger a quench is very high, in the Joule range $[5,6]$ instead of the $\mathrm{mJ}$ range for LTS. At operation closer to the critical temperature $T_{\mathrm{c}}$, the higher heat capacity of the winding also leads to a stable behavior, even though the temperature margin is smaller. For this reason, a quench in the classical sense of the term, meaning an unexpected localized 
heat deposition leading to catastrophic thermal runaway, is unlikely to happen in a HTS magnet.

However, if the conductor dissipates locally in the magnet, this high stability prevents the dissipation from propagating rapidly. Indeed, dissipative zone (DZ) propagation velocities in REBCO tapes are typically measured in the $\mathrm{cm}$ to $10 \mathrm{~cm} \mathrm{~s}^{-1}$ range [6]. In consequence, local damage can occur due to a local overstepping of the critical current leading to slowly growing dissipating regions (hotspots), with no obvious signal of the phenomenon being visible from the outside.

It seems incorrect to use the name quench when referring to such events in HTS magnets, and many studies have shown that the use of classical LTS magnet quench concepts, such as minimum quench energy and normal zone propagation velocity are not straightforward [7-9] in HTS applications as they are constantly varying with a number of parameters (temperature, current density, distance from initial dissipative spot, etc). In the following we will instead refer to our work as simply being the study of the occurrence and propagation of DZs.

Even assuming that the magnet design has no flaws (inhomegenous cooling, wrong evaluation of minimum $I_{\mathrm{c}}$, differential stress, etc) such local overstepping can be caused by local variations of the conductor performances. Indeed, present-day REBCO tapes show millimeter-scale local variations of their critical current $I_{\mathrm{c}}$ in the $10 \%$ to $20 \%$ range $[10,11]$. To overcome this problem, present-day HTS magnets are usually designed with large operation margins. Such margins make it possible to ensure the safe operation of practical magnets $[2,3]$, but reduce the performance that can be achieved when using them as the conductor is not used efficiently. Operating closer to the critical current, a larger volume fraction of reinforcement could be used, which would improve the mechanical properties further while reducing the cost.

One method successfully implemented to operate close to the limits of the conductor is to remove the insulation between turns [12], or to replace it with a co-winding of a normal conductor [13], so that the current can bypass the turns where dissipation is occurring. This method, however, has the disadvantage of affecting the magnet dynamic. The other method is to rely on a very sensitive detection system to detect the occurrence of DZs at a very early stage in order to initiate protection before the temperature rise becomes damaging. We tested this method successfully on R\&D pancake coils with engineering current densities up to $800 \mathrm{~A} \mathrm{~mm}^{-2}$ [14], and similar results were confirmed on test dipoles using HTS cables [15]. The use of very powerful heaters [16] to help the DZ propagate through the magnet (forcefully quenching it) was also tested successfully [2] even though there is a risk that the high thermal stress during such a protection event may have a negative effect on the coil durability.

Significant work has been conducted on the propagation of DZs, in most cases assumed to be pre-existing normal zones $[17,18]$. In this work, our aim is to develop a numerical tool to study the natural occurrence and propagation of DZs in a magnet, in the simple case of pancake-based solenoids with

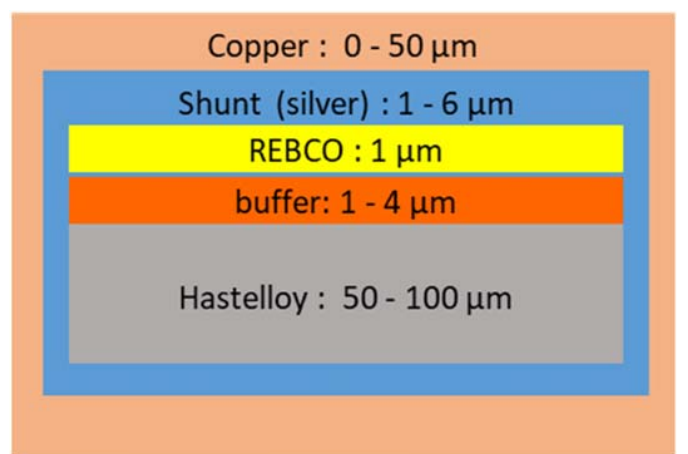

Figure 1. Configuration of a typical REBCO HTS tape.

a strong focus on the effect of local performance inhomogeneities along the conductor. There are ultimately two goals for this modeling effort:

- To quantify the requirements in terms of the early detection of DZs and in terms of a protection scheme in order to guarantee the survival of a given magnet.

- To evaluate the acceptability of conductor inhomogeneity distributions for a given magnet design with its detection/ protection system.

\section{Modeling approach}

REBCO-coated conductors have a multilayered structure. A $1-3 \mu \mathrm{m}$ thick REBCO layer is grown on a 50 to $100 \mu \mathrm{m}$ thick substrate usually made of Hastelloy ${ }^{\circledR} \mathrm{C} 276$, through a number of thin buffer layers. It is then surrounded by normal conducting layers for protection and electrical connection, typically $2-4 \mu \mathrm{m}$ of silver and $0-40 \mu \mathrm{m}$ of copper (figure 1 ).

\subsection{Electric model}

2.1.1. Electro-magnetic behavior. The REBCO material itself is a strongly anisotropic non-magnetic material, with a highly nonlinear $\mathrm{E}(\mathrm{J})$ constitutive equation. From an electromagnetic point of view, it can be described by the general equations (1) and (2).

$$
\begin{gathered}
\boldsymbol{B}=\mu_{0} \boldsymbol{H} \\
\boldsymbol{E}=\rho(\boldsymbol{J}, \boldsymbol{B}, T) \boldsymbol{J}
\end{gathered}
$$

with $\boldsymbol{B}$ denoting the magnetic flux density, $\mu_{0}$ the vacuum permeability, $\boldsymbol{H}$ the magnetic field, $\boldsymbol{E}$ the electric field, $\rho$ the electric resistivity, $\boldsymbol{J}$ the current density and $T$ the temperature. To model the nonlinear equivalent resistivity of the superconducting layer before reaching the critical temperature $T_{\mathrm{c}}$, a power-law model is often used (equation (3)).

$$
\rho_{S C}(\boldsymbol{J}, \boldsymbol{B}, T)=\left\{\begin{array}{cc}
\frac{E_{\mathrm{c}}}{J_{\mathrm{c}}(\boldsymbol{B}, T)}\left(\frac{\boldsymbol{J}}{J_{\mathrm{c}}(\boldsymbol{B}, T)}\right)^{n-1} T \leqslant T_{\mathrm{c}} \\
\rho_{N}(\|\mathbf{B}\|, T) & T>T_{\mathrm{c}}
\end{array} .\right.
$$

with $E_{\mathrm{c}}$ denoting the critical electric field set to $1 \mu \mathrm{V} \mathrm{cm}{ }^{-1}$, at which the critical current density $J_{\mathrm{c}}$ is defined. The $J_{\mathrm{c}}$ depends 


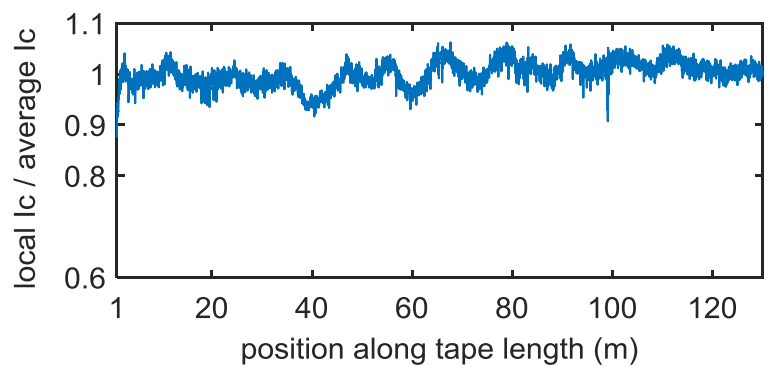

Figure 2. Local $I_{\mathrm{c}}$ variation around length average on a typical REBCO tape (SuperOx, $12 \mathrm{~mm}$ wide).

on local operating conditions of magnetic field (amplitude and orientation) and temperature, and also varies locally due to the conductor microstructure. $n$ is a dimensionless parameter generally ranging from 20 to 50 .

Over $T_{\mathrm{c}}$, the superconductor is in the normal state and its resistivity varies only with the temperature and magnetic field amplitude due to magneto-resistance.

Moreover, from an electrical point of view the superconducting layer is parallel with all the other conducting layers. The current is therefore shared between the superconducting layer and the other resistive layers when the former stops being non-dissipative.

2.1.2. Homogenization of the electro-magnetic problem. In principle, a detailed description of the electro-magnetic problem could be made at the local scale without homogenization, but this would necessitate dynamically evaluating the current distribution in the whole tape cross section, similar to what is done in HTS AC losses models such as are presented in $[19,20]$. This would not only be very intensive in terms of computing, but it would also require local values of critical current density in the cross section of the superconducting layer that are not practically available. On the other hand, full system quench modeling relying on large-scale homogenization in order to have a limited number of unknowns runs the risk of neglecting the key role of local inhomogeneities in the conductor performances. A lot of effort has been devoted to building numerical models to account for the coupled electro-magnetic-thermal behavior of HTS by simulating so-called 'quench' behavior with different levels of homogenization [9, 21-23].

Our approach is to homogenize the electro-magnetic problem at the level at which critical current characterization of the conductor is available. Indeed, REBCO tape critical current is usually measured continuously at the output of the production line, for example using a Tapestar ${ }^{\circledR}$ machine [24]. The result is a one-dimensional (1D) distribution of critical current in a fixed condition, which is with the conductor operating in a self-field and at $77 \mathrm{~K}$. The spatial resolution of such a system along the length depends on the speed of measurement but is typically in the $\mathrm{cm}$ range. The result of such characterization for a typical tape is shown below in figure 2 .

Then, for each element along the length that has a given critical current, calculation of current sharing between the

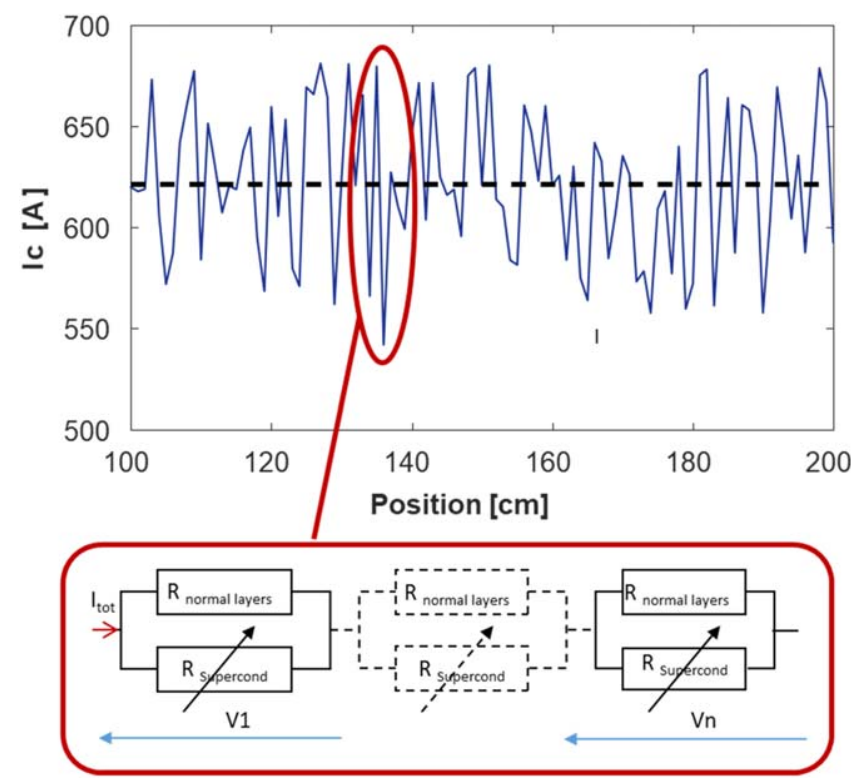

Figure 3. Lumped resistors circuit representing the tape, taking into account local $I_{\mathrm{c}}$ variations along the length.

layers will be used to obtain an equivalent resistance. From an electrical point of view, our model will therefore be $1 \mathrm{D}$ with a discretization no smaller than the characterization, which is around $1 \mathrm{~cm}$. The electro-magnetic model is thus simplified down to a simple electrical circuit that can be seen as seriesconnected lumped elements with nonlinear resistance (figure 3).

This same approach has already been used successfully for modeling the behavior of resistive superconducting fault current limiters [25, 26].

Of course in the case of a more complex conductor made of several tapes, a preliminary study of the conductor would be necessary, either experimentally or through modeling, to obtain the homogenized electric properties of the conductor per unit length.

\subsubsection{Accounting for the influence of the magnet field} distribution. In the case of a coil, the magnetic field is not the same everywhere along the conductor and its amplitude and orientation locally modify the critical current of the conductor. To account simply for that we use the concept of lift factor, which is defined as the ratio between the conductor critical current in a given temperature, orientation and temperature conditions $I_{\mathrm{c}}(T, B, \theta)$ and its $77 \mathrm{~K}$ self-field value $I_{\mathrm{c}_{\mathrm{s}}}$.f. $77 \mathrm{~K}$. This lift factor (figure 4) can be obtained through characterization of a short sample [27, 28], and can be assumed to be constant for a given conductor produced by a given supplier, at least as a first working hypothesis.

We therefore consider that the critical current variation along the length of the conductor is the product of the local lift factor by the local $I_{\mathrm{c}} 77 \mathrm{~K}$. s.f. performance of the studied conductor (4).

$$
\operatorname{Ic}\left(T_{o p}, B, \theta, x\right)=\operatorname{lift}(x) . \operatorname{Ic}_{77 K s . f}(x)
$$

where $x$ is the position along the length of the conductor forming the coil, and lift $(x)$ is the dimensionless multiplying 


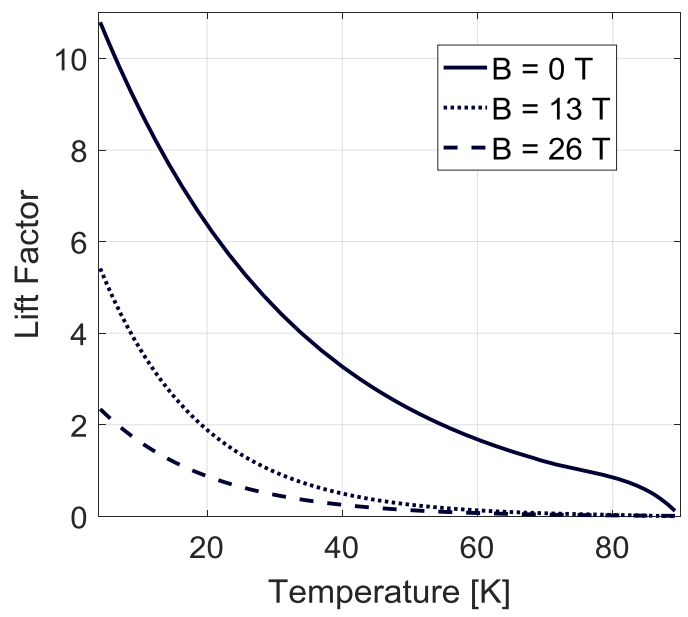

Figure 4. Lift factor data from measurements of a REBCO tape under various background fields [27].

factor accounting for the field amplitude and orientation. $T_{o p}$ is the local initial temperature (usually kept homogenous).

In order to obtain the $1 \mathrm{D}$ distribution of lift factor to be applied, a magnetostatic study of the full scale magnet is conducted beforehand to evaluate the local field amplitude and orientation along the whole length of the conductor forming the coil.

\subsection{Thermal problem}

2.2.1. Domain definition. The discretization of the thermal problem must be detailed enough to account for the effect of heat propagation in the magnet winding, including the effect of the very thin thermally-resistive layers of conductor insulation.

As our test case is pancake-based solenoids, a twodimensional (2D) model is sufficient as it accounts for the heat propagation both along the turns and from turn to turn, knowing that heat transfer from pancake to pancake is usually negligible. Out of plane exchanges can be added to account for the cooling system.

The studied area must be the one where the critical current is most likely to be locally overstepped which is the area in which the lift factor is the lowest.

Propagation of DZs in HTS coils remaining very local, the domain of study may also be limited to an angular portion. In such a case the initial pancake geometry can be represented by a rectangle (figure 5). This approximation is verified if the turn's radius is large enough compared to the studied length.

2.2.2. Thermal homogenization. For numerical modeling of the thermal problem, the thin-layered structure of REBCO tape is also an issue, as accounting for all layers of all turns would rapidly lead to a very large number of mesh elements with high aspect ratios.

In order to make it simpler, we homogenize the tape thermal properties. We choose to keep the surrounding insulation as a distinct layer on either side of the tape in order to keep the possibility of varying its thickness and to apply thermally-resistive interfaces between the turns.

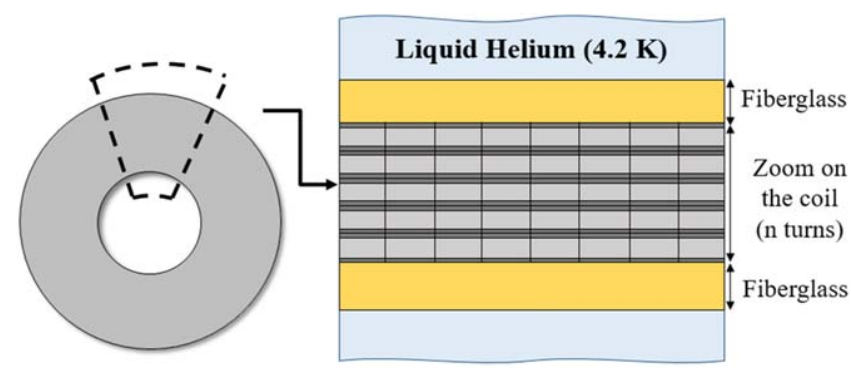

(a)

(b)

Figure 5. (a) Entire REBCO coil (viewed from above) and (b) focus on a small angular portion represented by a rectangle.

Concerning the thermal conductivity of the tape, the layered structure leads to an anisotropic thermal conductivity as the layers are in parallel along the conductor and in series across it (respectively, the horizontal and vertical direction in our simplified geometry figure 5). (5) and (6) give the equivalent longitudinal and transverse conductivities of an n-layered tape.

$$
\begin{aligned}
\lambda_{\text {long }} & =\frac{\sum_{n} \lambda_{n} \cdot e_{n}}{\sum_{n} e_{n}} \\
\lambda_{\text {trans }} & =\frac{\sum_{n} e_{n}}{\sum_{n} \frac{e_{n}}{\lambda_{n}}}
\end{aligned}
$$

where $\lambda_{n}$ and $\mathrm{e}_{n}$ are the thermal conductivity and the thickness of the $n$th layer.

An example of tape and insulation thermal properties is shown in figure 6; it corresponds to the tape and insulation specifications of the case studied in sections 3 and 4 .

\subsection{Coupling strategy}

In order to obtain a lighter and faster simulation tool, we use a co-simulation method, in which the two coupled models are solved sequentially for each time step (figure 7), following the so-called 'relaxation method' [29].

At each time step, the electrical model is launched first: it computes the current sharing between normal and superconducting layers for each of the 'blocks', that is, each element of the 1D electrical discretization of the conductor. In order to do so, it takes as input the blocks' temperatures at the previous time step. The heat generated in each block is then evaluated, and transferred to the thermal model.

The thermal model is launched in a second time, to calculate the heat propagation by solving the nonlinear transient heat equation using the finite element method (FEM) to update the conductor local temperature.

For this method to be valid, the time steps chosen by the solver for both models need not only comply with each model's stability requirements, they must also be made small enough that the error induced by the sequential solving is acceptable. 

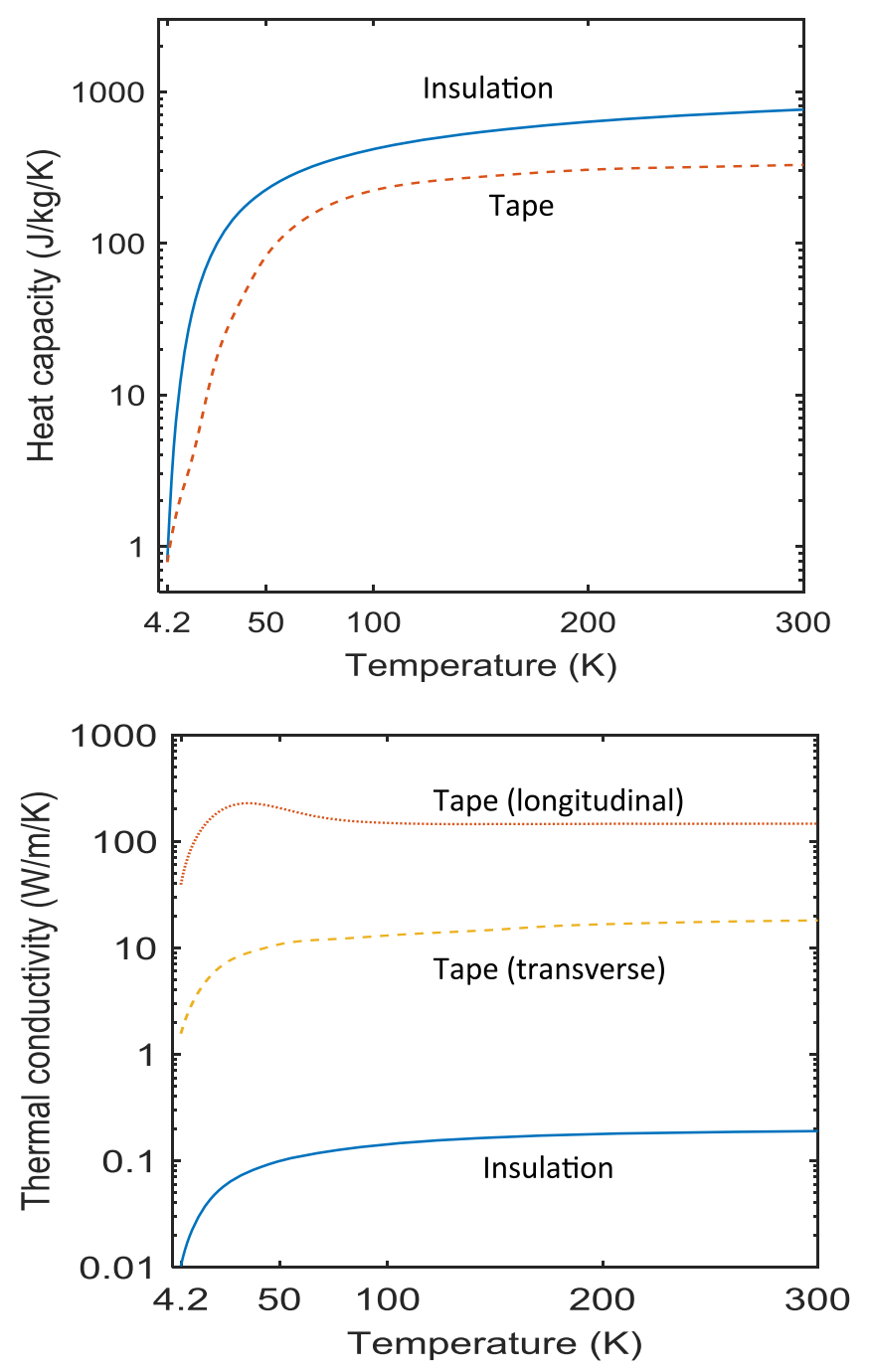

Figure 6. Top: specific heat capacities of insulation and tape. Bottom: thermal conductivity of insulation and tape (transverse and longitudinal directions).
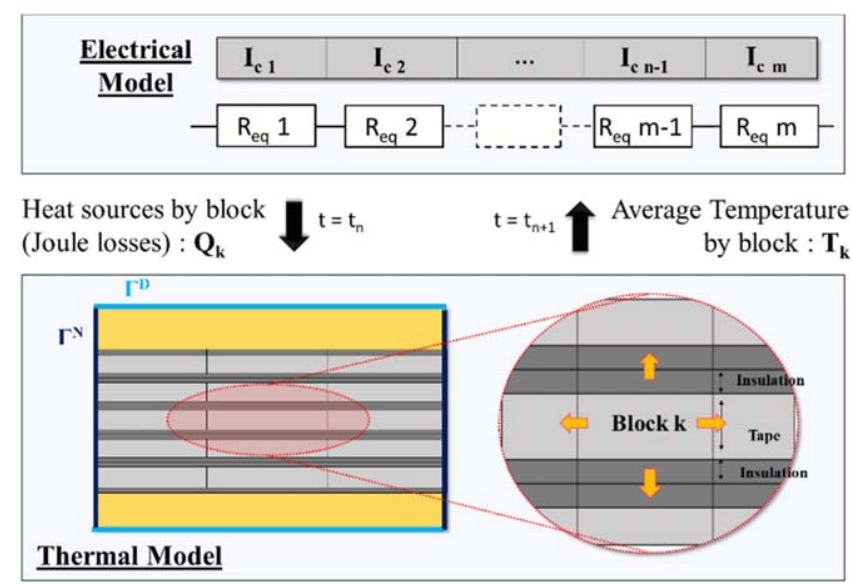

Figure 7. Coupling between electrical and thermal models.

\section{Implementation using $\mathrm{Comsol}^{\circledR}$ multiphysics}

We developed first a Comsol ${ }^{\circledR}$ implementation of that approach. It solves a 2D transient thermal problem by a FEM method. In the case where only conduction is considered the local heat equation is simply (7).

$$
\rho_{\mathrm{vol}} \mathrm{C}_{\mathrm{p}}(\mathrm{T}) \frac{\partial \mathrm{T}}{\partial \mathrm{t}}=\nabla \cdot(\lambda(\mathrm{T}) \nabla \mathrm{T})+\mathrm{Q}
$$

with $\rho_{\mathrm{vol}}$ denoting the density, $\mathrm{C}_{\mathrm{p}}$ the specific heat capacity, $\lambda$ the thermal conductivity and $\mathrm{Q}$ the heat source.

In our implementation, a different region is defined for each area corresponding to a conductor block in the electrical model. Each of these regions is defined as a heat source, whose value is evaluated prior to each time step by means of an external function.

\subsection{Current-sharing function}

This external function performs the role of the electrical model. It is evaluated separately for each block and takes as input the average of the temperature on the block, the local field amplitude and orientation, the local $I_{\mathrm{c}} 77 \mathrm{~K}$ s.f. performance, and the applied current. It first evaluates the local lift factor and derives the local $I_{\mathrm{c}}$. It then evaluates the dissipation by solving the current sharing between the different conductor layers.

For evaluating this current sharing, the layers are seen as two resistances in parallel. On one hand, there is the superconducting layer with its power-law equivalent resistance $\mathrm{R}_{\mathrm{SC}}(\mathbf{J}, \mathbf{B}, \mathrm{T})$. On the other hand there are all the normal conducting layers with an equivalent resistance $R_{\text {shunt }}(T)$ that only depends on the temperature (and possibly the field in some cases).

By equalizing both resistive voltages, we obtain (8) which gives the ratio of current $\beta$ flowing through the superconducting layer.

$$
\beta^{\mathrm{n}}+\frac{\left(\mathrm{I}_{\mathrm{c}}(\mathbf{B}, \mathrm{T})\right)^{\mathrm{n}}}{\mathrm{L}_{\mathrm{Block}} \mathrm{E}_{\mathrm{c}}} \mathbf{R}_{\text {shunt }} \beta-\frac{\left(\mathrm{I}_{\mathrm{c}}(\mathbf{B}, \mathrm{T})\right)^{\mathrm{n}}}{\mathrm{L}_{\mathrm{Block}} \mathrm{E}_{\mathrm{c}}} \mathbf{R}_{\text {shunt }}=0
$$

$\mathrm{L}_{\text {Block }}$ corresponds to the block length. This n-order polynomial equation has $\mathrm{n}$ roots but it can be easily demonstrated that only one root has a real positive value.

The equation is solved iteratively, for example using the Newton-Raphson algorithm. We then deduce the heat generated by each block $\mathrm{k}$ at a given time $\mathrm{t}$ : $\mathrm{Q}_{\mathrm{k}}(\mathrm{t})$.

\subsection{First results}

3.2.1. Test case presentation. This tool was applied to the simulation of the occurrence and propagation of DZs in a simple test case. The considered geometry is a $10 \mathrm{~cm}$ long, 30-turns thick portion of a pancake, approximated as a rectangle. This pancake is part of a magnet that generates a field proportional to the applied current. This field is considered homogenous on the domain of study, at $20 \mathrm{~T}$ for $521 \mathrm{~A}$, with an orientation of $20^{\circ}$ from the tape surface.

The initial temperature is $4.2 \mathrm{~K}$. The cooling is represented by a $1 \mathrm{~mm}$-thick layer of fiberglass on the top and bottom of the domain as shown on figure 7, with an imposed temperature of $4.2 \mathrm{~K}$ on the outer boundaries $\Gamma^{D}$. The side boundaries $\Gamma^{N}$ are considered adiabatic. The mesh for the FEM solving of the thermal transient problem is mapped, with three elements in the tape thickness, one on the 


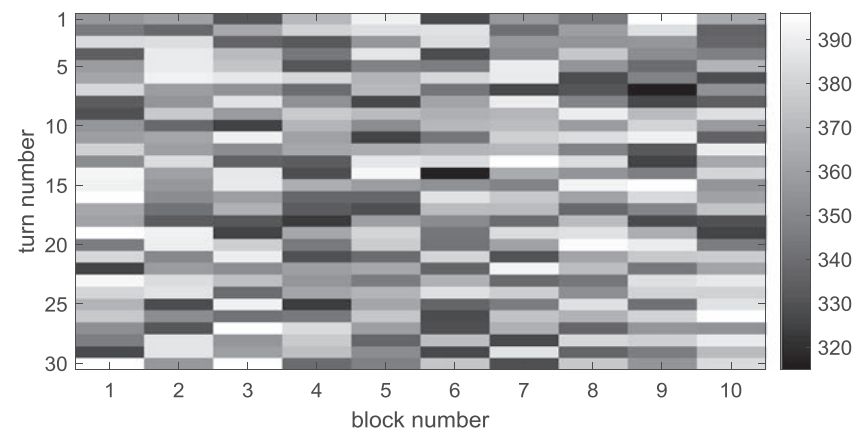

Figure 8. Distribution of $I_{\mathrm{c}}$ at $77 \mathrm{~K}$ s.f. on the domain (A).

Table 1. Tape properties in a model test case.

\begin{tabular}{ll}
\hline \multicolumn{2}{c}{ Conductor geometry } \\
\hline Silver & $2 \mu \mathrm{m}$ \\
Copper & $50 \mu \mathrm{m}$ \\
REBCO & $1.5 \mu \mathrm{m}$ \\
Substrate & $90 \mu \mathrm{m}$ \\
Buffer & $2 \mu \mathrm{m}$ \\
Insulation (surround) & $2 \times 15 \mu \mathrm{m}$ \\
Total thickness & $\mathbf{1 7 5 . 5} \boldsymbol{\mu m}$ \\
Tape width $\quad \mathbf{1 2 ~ \mathbf { m m }}$ \\
\multicolumn{1}{c}{ Conductor performance } \\
Average $I_{\mathrm{c}} 77 \mathrm{~K}$ s.f. & $332.55 \mathrm{~A}$ \\
$I_{\mathrm{c}}$ std dev. & $6.96 \%$ \\
Lift factor at $4.2 \mathrm{~K}, 20 \mathrm{~T}, 20^{\circ}$ & 2.02 \\
Lift factor at $20 \mathrm{~K}, 20 \mathrm{~T}, 20^{\circ}$ & 1.037 \\
\hline
\end{tabular}

insulation thickness, and five in the fiberglass thickness. Along the length, 100 elements are used.

For the electrical model, the $10 \mathrm{~cm}$ length is divided into ten blocks, which leads to a problem consisting of 300 elements connected in series.

The tape geometry and critical current properties are summarized in table 1 . The tape $I_{\mathrm{c}}$ variation was randomly generated in a $\pm 15 \%$ window around average, the resulting distribution on the domain is given in figure 8 .

The initial current in the study is taken at least $50 \mathrm{~A}$ under the smallest critical current value of the tape so as to be far enough from the transition initiation to initialize the domain with a homogeneous temperature of $4.2 \mathrm{~K}$, that is around $500 \mathrm{~A}$ in this case. The charging rate is $10 \mathrm{~A} \mathrm{~s}^{-1}$. The total voltage is monitored and discharge is triggered if the voltage oversteps a threshold whose value can be adjusted. The discharge is made at a constant $40 \mathrm{~A} \mathrm{~s}^{-1}$ rate.

3.2.2. First results and discussion. Figure 9 illustrates the dynamic of the study, in the case of a $500 \mu \mathrm{V}$ threshold value. In the first phase there is no dissipation as the current is well below the critical current at the weakest point of the conductor. In principle this phase is very long, that is why the simulation starts with a non-zero initial current. The variations in that phase are very slow so that the adaptive time-stepping method in $\mathrm{Comsol}^{\circledR}$ tends to increase the time steps considerably. However, when the minimum critical current of the domain is reached and

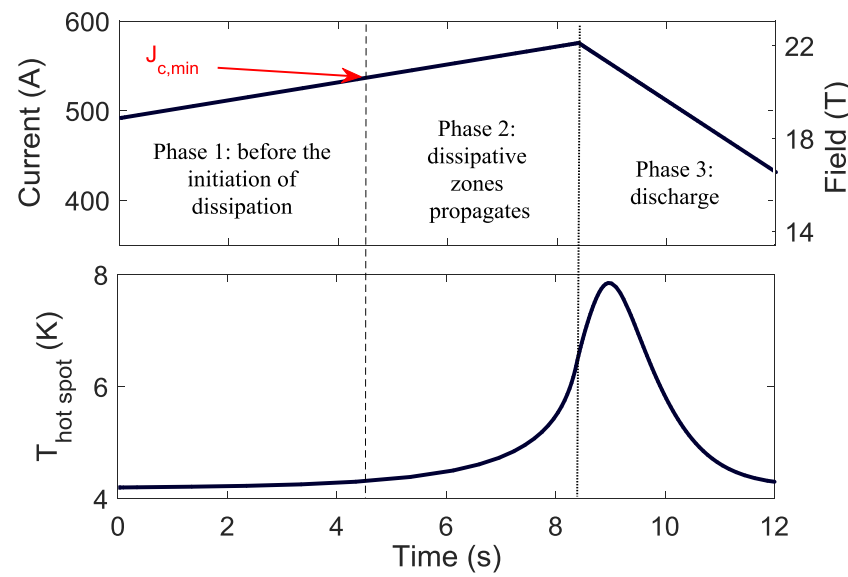

Figure 9. Temperature dynamic (bottom) divided into periods and the corresponding current and resulting magnetic field (top).

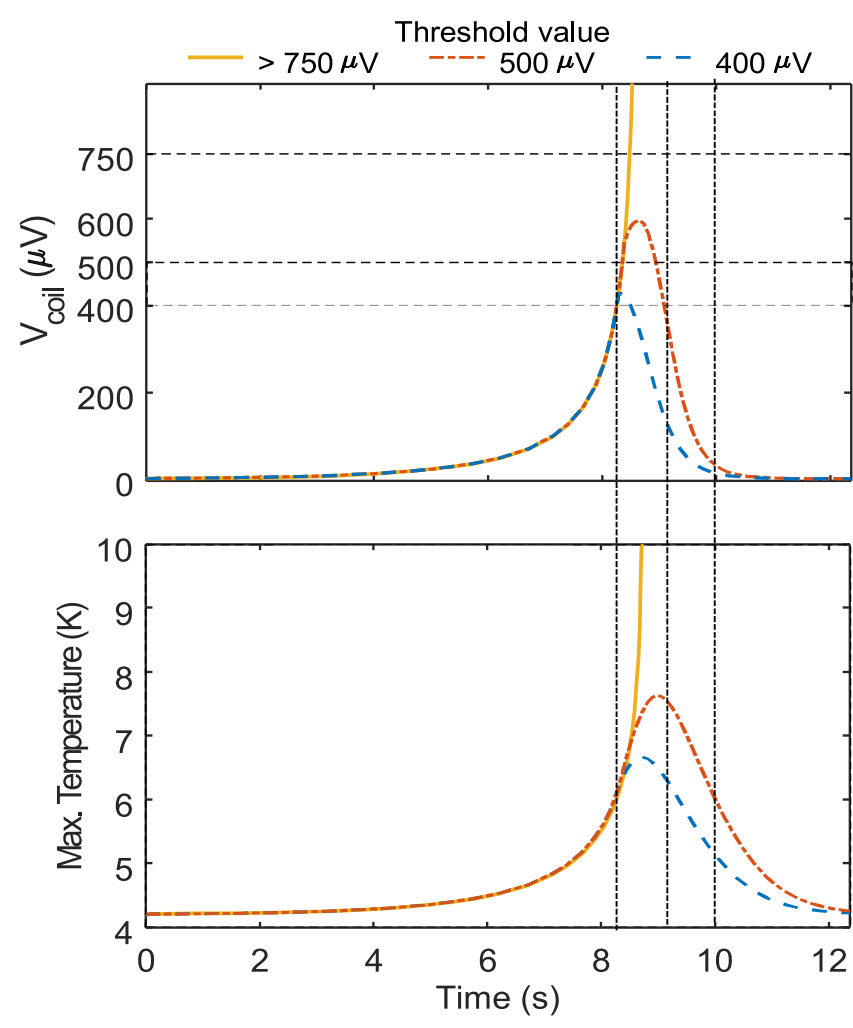

Figure 10. Evolution of the dissipation voltage and the temperature for different protection threshold values.

temperature starts to increase, the dynamic becomes suddenly very fast. There is a risk of missing the onset of that second phase if the time steps were extended too much, due to our sequential solving approach. In order to prevent that, a very low relative tolerance is used for the nonlinear solver of the thermal problem (lower than 1e-6). Phase three starts when the threshold is reached: the current starts to decrease.

This discharge may lead to a recovery of the non-dissipative state or a destructive runaway. It depends both on the threshold value and the discharge dynamic. A parametric study was conducted to quantify the effect of the threshold value (figure 10), the discharging being kept at a constant $40 \mathrm{~A} \mathrm{~s}^{-1}$. 


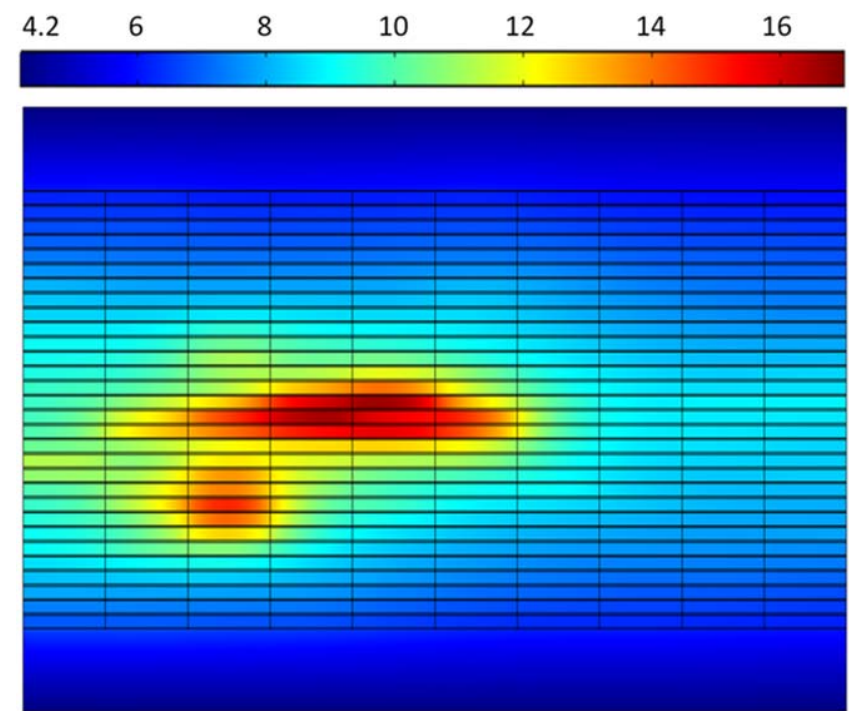

Figure 11. Temperature distribution $(\mathrm{K})$ at $\mathrm{t}=8.7 \mathrm{~s}$ for the case without protection discharge (peak temperature is $17 \mathrm{~K}$ ).

With a $400 \mu \mathrm{V}$ threshold, the discharge is triggered at $8.25 \mathrm{~s}$, that is under $574.3 \mathrm{~A}$. The maximum temperature is reached at $8.7 \mathrm{~s}$. In the meantime the current has only decreased to $556 \mathrm{~A}$. If the threshold is $500 \mu \mathrm{V}$ the discharge is triggered $0.1 \mathrm{~s}$ later, and thus $1 \mathrm{~A}$ higher. The maximum temperature is reached at $9 \mathrm{~s}$ and goes up to $7.8 \mathrm{~K}$ (under 550 A). Finally, if the threshold is set to $750 \mu \mathrm{V}$, the discharge is triggered only $0.23 \mathrm{~s}$ later and still this is too late to obtain a recovery.

In a real case, the detection system delay and the protection switching time may very well add a similar delay. This tends to prove that to reliably protect the coil, the threshold value must be set significantly lower, in the $100 \mu \mathrm{V}$ range, enough so that the available time for reacting is higher than the second.

Such a low threshold value is difficult to reach practically. Even though we achieved it on small systems [14], in all likelihood it would be impossible to reach reliably on a full scale magnet. It must however be observed that the presented study is on a very small portion of winding, about $6 \mathrm{~cm}^{3}$. In a real case many other areas of the magnet would be submitted to similar operation conditions, so that the required threshold value would be multiplied as well.

Figure 11 shows the temperature distribution at $8.7 \mathrm{~s}$ for the $750 \mu \mathrm{V}$ case. Two localized neighboring hotspots appear. Comparing that result to figure 8 , it seems that predicting the areas where DZs will appear is not straightforward.

\subsection{Limitations in the use of an external function in $\mathrm{Comso}^{\circledR}$}

As mentioned earlier, the $\mathrm{Comsol}^{\circledR}$ implementation is essentially a transient thermal model, in which at the beginning of each time step an external function performs the role of the electrical model. The drawback of such a method is that the time stepping is governed by the thermal model only. There is no way for the electrical model to reject a time step if the variation during it is too large. This issue is solved practically by enforcing very tight tolerances on the thermal model, but the use of external functions in $\mathrm{Comsol}^{\circledR}$ has additional limitations.

Firstly, such a function does not store past results, which means that the iterative solver of the current sharing cannot be initialized differently at each time step. The Newton-Raphson algorithm, whose convergence depends drastically on initialization, is therefore not efficient in this implementation. A basic iterative scheme with over-damping [25] becomes faster on average, but still requires several tenths of iterations.

Secondly, such a function can only output a scalar. The current-sharing function is thus called as many times as there are blocks, each calculating the heat generated on one block independently. There is no way during the functions execution to get the total equivalent resistance of the seriesconnected circuit and therefore no possibility to evaluate the current with circuit-based equations. The current needs to be imposed, as well as the field.

\section{Implementation in a versatile modeling environment}

\subsection{Motivation}

In order to have more flexibility especially in the electrical model, we implemented the same modeling approach in a multi-level and multi-method simulation platform called MIPSE, developed in Grenoble by the G2ELab [30]. Originally focused on low and average frequency computational electromagnetism and electrical circuits, we extended it by introducing a nonlinear transient thermal formulation allowing it to solve coupled electro-magnetic and thermal problems. The main advantage is that it allows control on the numerical methods that are used and offers a higher flexibility than commercial software for creating coupling between models. It also makes it possible to test unusual adaptive time-stepping methods.

Moreover, in practical experiments the variations on the coil voltage are not only due to DZs, but also on complex dynamic effects of current density distribution. These phenomena must be understood in order to discriminate dangerous localized dissipation from distributed low-level dissipation and/or inductive voltages due to transient effects. An efficient modeling tool to study such phenomena is simultaneously being developed in the MIPSE platform using integral methods [31]. Our objective is eventually to interface both electro-thermal and electro-magnetic models in order to develop an integrated multiphysics multi-purposed simulation tools for HTS applications.

\subsection{Upgraded electrical circuit model}

In this implementation, the circuit-based electrical model can represent the charge and discharge circuit of the HTS coil (figure 12), including the switch dynamic. 


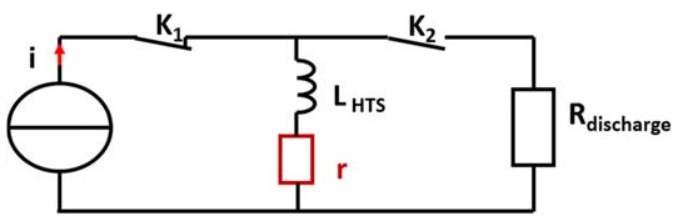

Figure 12. Charge and discharge circuits of a standalone HTS magnet.

Table 2. Convergence improvement using prediction.

\begin{tabular}{lll}
\hline & $\begin{array}{l}\text { Standard } \\
\text { initialization }\end{array}$ & Prediction \\
\hline Computation time & $4129 \mathrm{~s}$ & $3087 \mathrm{~s}$ \\
& $(1 \mathrm{~h} 8 \min 49 \mathrm{~s})$ & $(51 \mathrm{~min} 27 \mathrm{~s})$ \\
Total number of nonlinear & 747 & 489 \\
iterations & & \\
\hline
\end{tabular}

It is also possible to study the interaction of the HTS coil with another coil in order to accurately represent the discharge dynamics of a hybrid LTS/HTS magnet, for example.

For solving the electrical model non-linearity, a NewtonRaphson algorithm with an initialization at the previous time step is being used. The number of iterations required is usually very low, often below three.

\subsection{Thermal model}

The transient thermal FEM formulation is a new development in the MIPSE platform. It deals with both the non-linearity of the specific heat capacities and the thermal conductivities which makes solving time-consuming, especially considering that the MIPSE platform is not optimized for processing speed but rather for customization. We implemented two different refinements to improve the computation efficiency. The first one aims at reducing the Newton-Raphson iteration number needed to converge by initializing through a prediction of the next solution. The second one intends to find an optimized sequence for the time discretization while satisfying the precision required.

\subsubsection{Newton-Raphson convergence improvement. As} mentioned earlier, when using the Newton-Raphson algorithm the choice of the initial condition for the iterative process has a strong influence on the convergence speed. The easiest way to initialize is to take the solution at the previous time step, as was done for the electrical model. To get even faster convergence, in the thermal model a predictive algorithm is used for the initialization at each time step based on previous solutions. It uses a first-order polynomial interpolation based on a combination of forward and backward difference methods. Equation (9) expresses the predicted solution $u_{n+1}$ at the next time $t_{n+1}$ in the most general case, i.e. an adaptive time step.

$$
u_{n+1}=u_{n}\left(1+\frac{\Delta t_{n+1}}{\Delta t_{n}}\right)-\frac{\Delta t_{n+1}}{\Delta t_{n}} u_{n-1}
$$

with $\Delta t_{k}$ being the time step at the $k$ th iteration.

Table 2 shows the computation performances of the prediction used to initialize Newton-Raphson compared to a
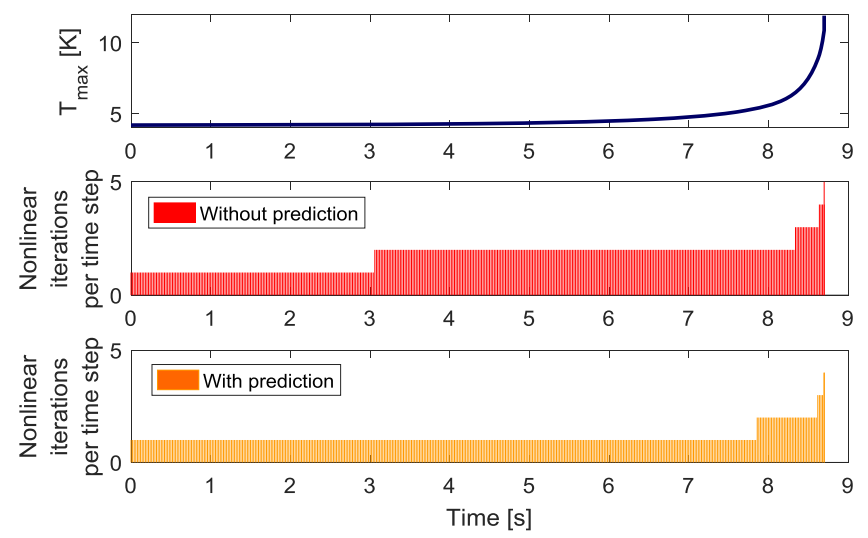

Figure 13. Nonlinear iteration number per time step with and without prediction along with the maximum temperature evolution (thermal runaway case).

more standard approach where the initialization is made by the previous solution.

Both tests are made with a fixed time step of $20 \mathrm{~ms}$. The problem being solved is identical in all details to the one tested with the Comsol ${ }^{\circledR}$ implementation in section 3.2.1.

At the very beginning, both methods are equivalent but quickly the predictive method seems to evaluate the next solution accurately enough to decrease the total number of nonlinear iterations over significant periods (figure 13). The prediction tool saves around $25 \%$ of computation time by accurately anticipating the solution at the next time step.

However, at the very end the Newton-Raphson iteration number still increases, because the time step is kept constant even though the non-linearity becomes worse. This illustrates the interest of adaptive time stepping.

4.3.2. Adaptive time-stepping methods: the interest of an integral controller. For the time discretization of the thermal model, a backward difference method is adopted to ensure stability. No numerical restrictions are imposed on the stepsize values, although they are limited by a lower and an upper boundary defined by the user.

In order to minimize the computation time while meeting the required accuracy, the use of a classical state-variable variations control method was tested first (method 1). This method aims at limiting the step size in case of quick variation of the state variable (here: the temperature) in order to ensure a good accuracy of the result. An upper limit allowing for the maximum temperature variation $\Delta T_{\max }$ from one iteration to the other is defined. If the computed variation exceeds the assigned value, the solution is rejected and the time step is decreased by a constant factor (for instance it is divided by two). On the other hand, if the computed variation is far below the limit, the next time step is increased (multiplied by a fixed coefficient greater than 1).

Figure 14 shows the procedure applied for one time-step computation. The solution is computed with successively smaller time steps until the variation criterion is respected. The main drawback of this method is the tuning of the two coefficients $\alpha$ and $\beta$, depending on the dynamic of the system: 


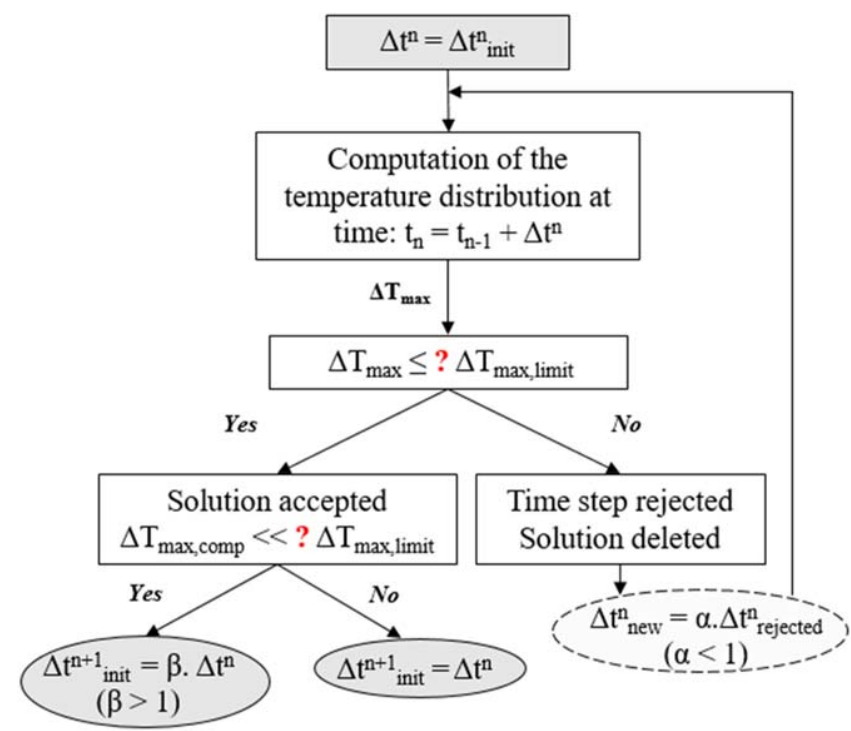

Figure 14. Adaptive time stepping: method 1 based on an upper limit of the state-variable variations.

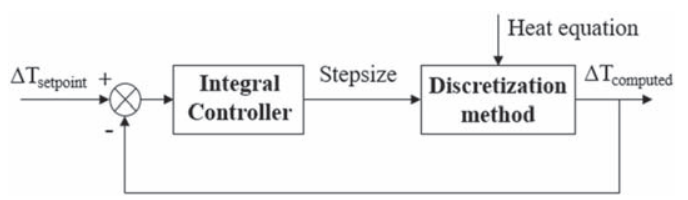

Figure 15. Feedback loop with integral controller applied to the heat equation.

the smaller $\alpha$ is, the more likely the next time-step solution will be far below the limit meaning that the step size began unnecessarily small.

A promising alternative option for adjusting the time-step size is to look for tools from the automatic control theory to regulate a specific quantity (method 2). Combining the automatic control approach with numerical methods leads to smoother step-size sequences [32].

In [33], Söderlind presents some developments of stepsize adaptability algorithms based on techniques from linear feedback control theory. We decided to test the integral controller: the step size computed is a weighted sum of all past control errors. The feedback loop with a control on the maximum temperature variation between two time steps is described in figure 15.

In this approach, the process to be regulated is the discretization method and its transfer function is expressed in the Z-transform domain by a gain $k$ whose value depends on the order of convergence of the method. The heat equation's properties are seen as a perturbation. The maximum temperature variation computed $\Delta T_{\text {computed }}$ is compared to the set point value $\Delta T_{\text {set point }}$ and the next time step is computed according to the error $\varepsilon=\Delta T_{\text {set point }}-\Delta T_{\text {computed }}$, thanks to the integral control described in the time domain by (10).

$$
\Delta \mathrm{t}^{n+1}=\left(\frac{\gamma \cdot \Delta T_{\text {set point }}}{\Delta T_{\text {computed }}^{n}}\right)^{1 / \mathrm{k}} \Delta \mathrm{t}^{n}
$$

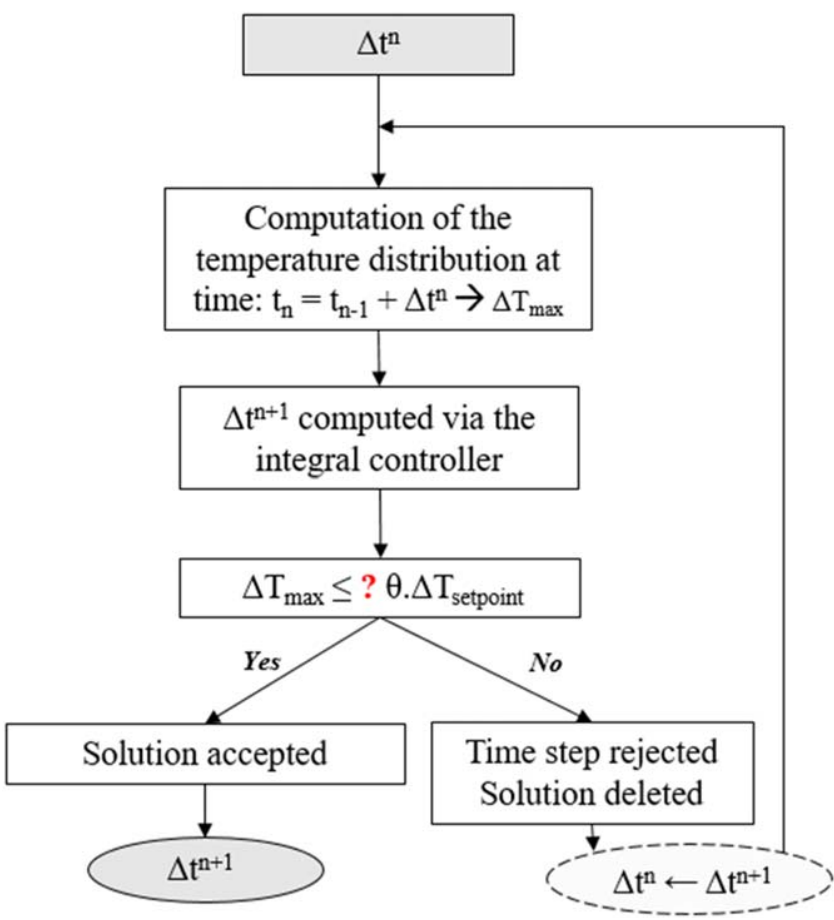

Figure 16. Adaptive time stepping: method 2 based on an integral controller.

Table 3. Computation time reduction using an integral controller for adaptive time stepping.

\begin{tabular}{lcc}
\hline & Method 1 & Method 2 \\
\hline Computation time & $2006 \mathrm{~s}$ & $1266 \mathrm{~s}$ \\
& $(33 \min 26 \mathrm{~s})$ & $(21 \operatorname{min~} 6 \mathrm{~s})$ \\
Total number of time steps & 176 & 114 \\
Rejected time-steps number & 12 & 8 \\
Total number of nonlinear iterations & 312 & 201 \\
\hline
\end{tabular}

where the exponent $n$ (or $n+1$ ) denotes the iteration number and $\gamma$ is a safety factor fixed at 0.8 to reduce the risk of step rejection [32]. Similarly, we define a coefficient $\Delta>1$ in such a way that if $\Delta T_{\text {computed }}^{n}>\delta . \Delta T_{\text {set point }}$, the step is rejected: the solution is deleted and computed again with a smaller time step. This approach is summarized in figure 16.

The two methods were applied to the test case of section 3.2.1, also using the prediction method introduced in section 4.3.1. $\Delta T_{\max }$ is set at $0.1 \mathrm{~K}$ : it represents an upper limit for the first method whereas it is considered as a set point for the second one. The minimum and maximum stepsize values are $0.1 \mathrm{~ms}$ and $1 \mathrm{~s}$ respectively. The $\delta$-coefficient of method 2 is set to 1.2 .

Computation performances of both methods are detailed in table 3. As expected, the second method exhibits better performances thanks to its integral action: it accurately anticipates the dynamic of the problem based on what happened previously. The step-size sequence using method 1 varies severely (figure 17) as soon as the limit is reached. The step-size sequence of method 2 varies continuously (figure 18) which results in less time steps but also a smaller rejection rate. 

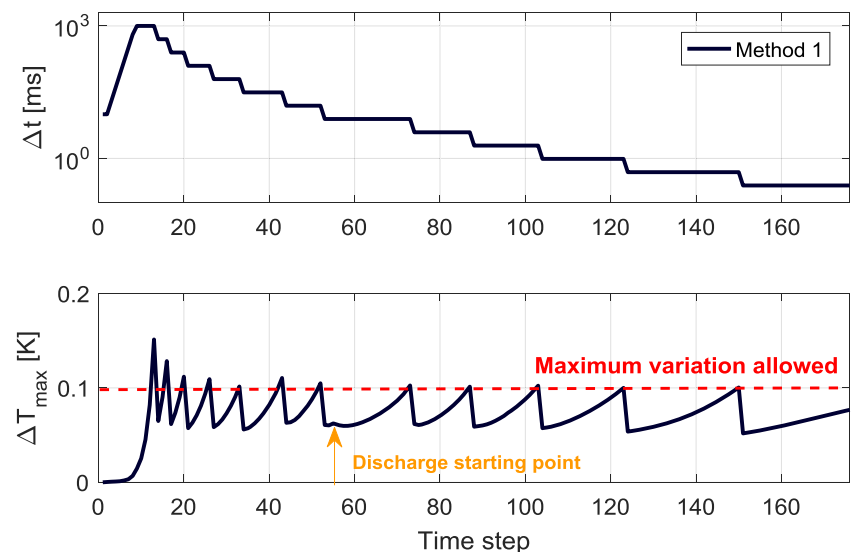

Figure 17. Step-size sequence (top) and successive values of the monitored quantity $\Delta T_{\max }$ (bottom): method 1 .
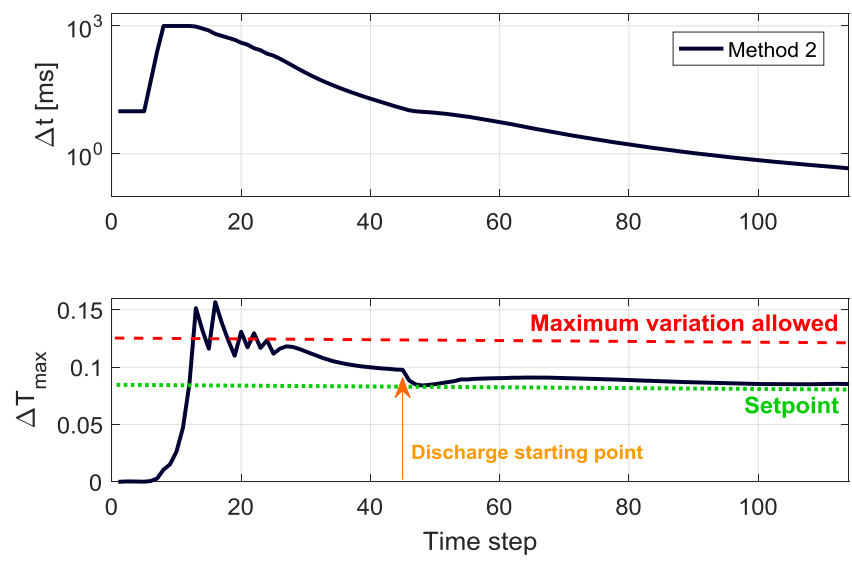

Figure 18. Step-size sequence (top) and successive values of the monitored quantity $\Delta T_{\max }$ (bottom): method 2 .

\subsection{Validation of the MIPSE implementation}

The integral controller combined with the prediction method allows a reduction of the computation effort while ensuring the same accuracy as a small fixed time step by choosing appropriated step sizes. The computation time is divided by more than three (from $1 \mathrm{~h} 8 \mathrm{~min}$ to $21 \mathrm{~min}$ ). The evolution of the maximum temperature is compared in figure 19 between the reference result obtained with Comsol ${ }^{\circledR}$ and both the constant time step and adaptive time-step versions in MIPSE, showing an almost perfect match, in the case with the threshold value at $750 \mu \mathrm{V}$ (no recovery), even though the implementation is totally different.

The computed dissipation voltage with the three threshold values plotted in figure 10 for the $\mathrm{Comsol}^{\circledR}$ implementation is compared in figure 20 with the MIPSE variable time-step implementation on a narrower timespan to discern the differences. The results are also in very good agreement but a small difference can be seen, especially during the discharge, where the MIPSE model's dissipation voltage is decreasing faster. The most likely explanation for this is that the lift factor interpolation method in both implementations is not the same. This has a more prominent effect during the

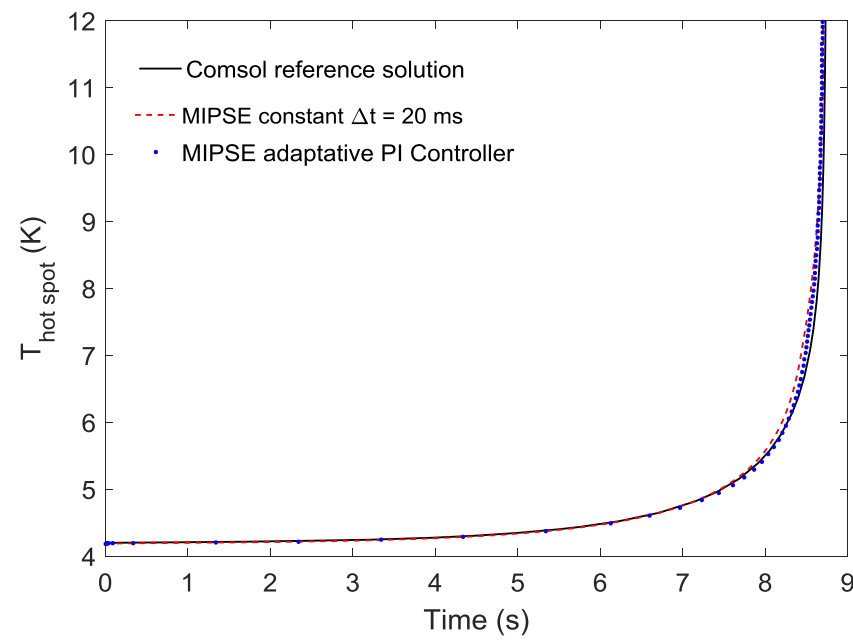

Figure 19. Maximum temperature evolution computed with $\mathrm{Comsol}^{\circledR}$ (reference) and with both the fixed time-step and adaptive time-step version of MIPSE.

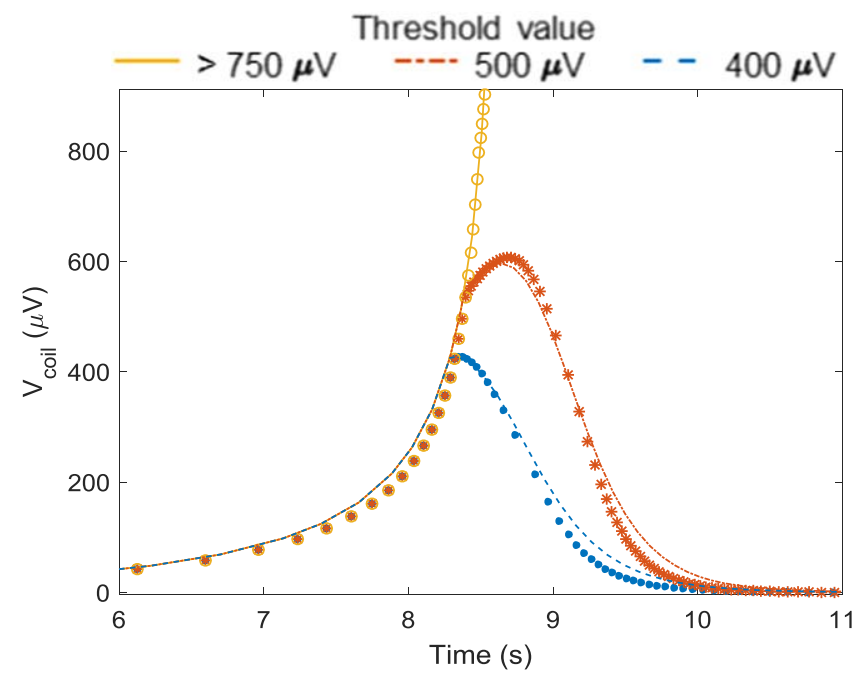

Figure 20. Dissipation voltage computed with Comsol ${ }^{\circledR}$ (lines) and with the adaptive time-step version of MIPSE (points) for various threshold values.

discharge as the current, and therefore the field, varies four times faster than during the charge.

Finally, the temperature distribution obtained using MIPSE is compared to the one already obtained in $\mathrm{Comsol}^{\circledR}$. In figure 21 the two models' results were rendered in the same color scale.

\section{Results discussion}

The test case considered was kept intentionally simple in order to make it easy to reproduce. However, some practical conclusions can be drawn. First, the results confirm that the existence of a distribution of inhomogeneity must be taken into account to efficiently protect a HTS coil in case of quench. This can be seen in the temperature distribution in figure 11 , where even though the dissipation is localized, 

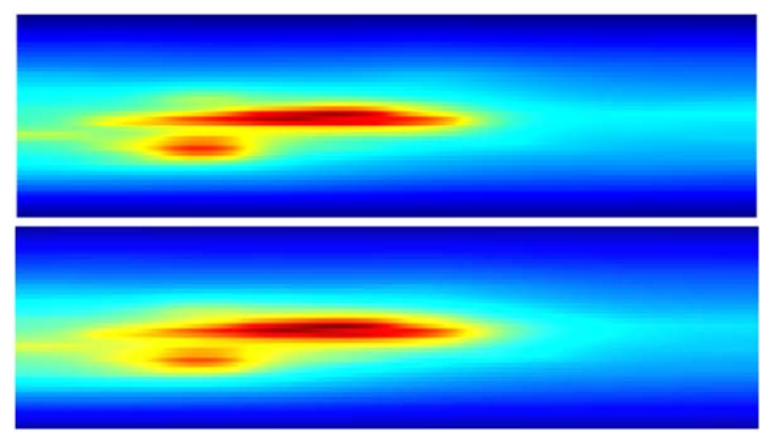

Temperature $[\mathrm{K}]$

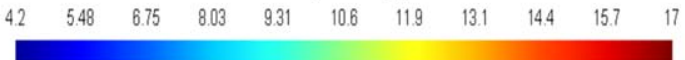

Figure 21. Temperature distribution $(\mathrm{K})$ at $\mathrm{t}=8.7 \mathrm{~s}$ for the case without protection discharge (peak temperature is $17 \mathrm{~K}$ ). The upper figure is $\mathrm{Comsol}^{\circledR}$, the lower is MIPSE.
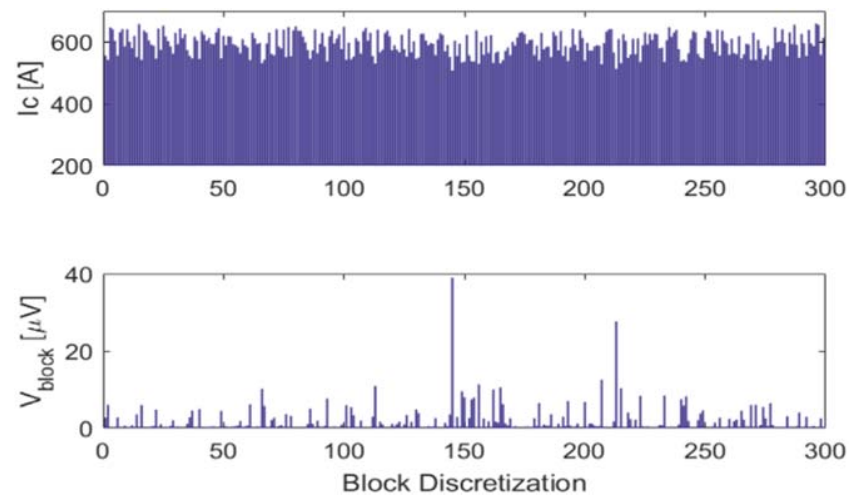

Figure 22. Voltage repartition and critical current over the electrical discretization (blocks) for the $500 \mu \mathrm{V}$ threshold value; taken when the protection has just been triggered.

there is significant diffusion, including between turns. Figure 22 shows it from another point of view. The total voltage is distributed along the block discretization of the electrical part.

The weakest block in terms of $I_{\mathrm{c}}$ at the $77 \mathrm{~K}$ self-field is indeed the place where the dissipation is highest. However, its voltage at the moment of triggering the discharge represents only $7.3 \%$ of the total voltage. Other blocks with originally weak $I_{\mathrm{c}}$ have also started to dissipate, as well as other neighbors due to thermal diffusion.

It means that a method relying on the adiabatic study of a single localized weak point to define protection strategies would be over-pessimistic.

As mentioned before, the threshold values required to allow recovery are in the $100 \mu \mathrm{V}$ range if one allows a realistic delay for the protection triggering. But the studied domain is only $6 \mathrm{~cm}^{3}$. Assuming that all the areas of the magnet submitted to similar operation conditions behave similarly, the required threshold would be multiplied by a factor of 10 or more. Discriminating a $\mathrm{mV}$ range dissipation signal from the noise is challenging but feasible. Protecting a full magnet from the consequence of one single conductor defect, leading to the occurrence of a single DZ would be very difficult. In consequence, conductor lengths with a single prominent defect should not be accepted for winding, even if such defect performance is above the expected nominal current.

Finally, it was observed in practical experiments that the transient distribution of the current in the conductor can induce variations of the voltage across the coil in the $\mathrm{mV}$ range, under constant current ramps even for small coils [14]. The dynamic of such signals must be understood in order to discriminate the appearance of such voltages from DZ occurrence.

\section{Conclusion}

An electro-thermal model has been proposed to simulate the apparition and propagation of DZs in REBCO HTS coil. Even though the present work assumes isolation between turns, the model can also be applied to non-insulated or metal-asinsulation coils to study behavior just before the onset of current sharing between turns, which is interesting in terms of predicting their operation limits. Two implementation were made, one in the commercial software $\mathrm{Comsol}^{\circledR}$ and another in the in-house developed MIPSE platform. The former has the advantage of being very easy to use, while the latter has more flexibility and will be later integrated with other modeling tools focused on other aspects of REBCO coil designs, such as field stability, for example. The results obtained in the two implementations are in very good agreement. In the MIPSE platform, the use of a prediction for the nonlinear initialization along with an adaptive time-stepping method with an integral controller action makes it possible to significantly reduce the computation time. Finally, the results obtained on a small $10 \mathrm{~cm}$ long 30-turns thick portion of pancake illustrate the importance of taking into account the local inhomogeneity of the conductor, as well as the thermal propagation in the winding. Applied to a specific magnet design, it is our hope that this model will allow us to determine detection threshold and protection schemes ensuring safe operation. It could also be a means of setting acceptability criterion for the inhomogeneity of REBCO conductor lengths.

\section{ORCID iDs}

Arnaud Badel (iD https://orcid.org/0000-0002-5273-2612

Blandine Rozier (ib https://orcid.org/0000-0002-2945-8918

Gérard Meunier (1) https://orcid.org/0000-0001-6424-1653

\section{References}

[1] Xu A et al $2017 \mathrm{Je}(4.2 \mathrm{~K}, 31.2 \mathrm{~T})$ beyond $1 \mathrm{kA} \mathrm{mm}{ }^{-2}$ of a $\sim 3.2 \mu \mathrm{m}$ thick, $20 \mathrm{~mol} \% \mathrm{Zr}$-added MOCVD REBCO coated conductor Sci. Rep. 76853 
[2] Weijers H W et al 2016 Progress in the development and construction of a 32-T superconducting magnet IEEE Trans. Appl. Supercond. 26 1-7

[3] Awaji S et al 2017 First performance test of a 25 T cryogenfree superconducting magnet Supercond. Sci. Technol. 30 065001

[4] Yoon S, Kim J, Cheon K, Lee H, Hahn S and Moon S-H 2016 $26 \mathrm{~T} 35 \mathrm{~mm}$ all- $\mathrm{GdBa}_{2} \mathrm{Cu}_{3} \mathrm{O}_{7-\mathrm{x}}$ multi-width no-insulation superconducting magnet Supercond. Sci. Technol. 29 04LT04

[5] Miyoshi Y et al 2015 Performance tests of prototype high-field HTS coils in Grenoble IEEE Trans. Appl. Supercond. 25 1-5

[6] Casali M, Breschi M and Ribani P L 2015 Two-dimensional anisotropic model of YBCO coated conductors IEEE Trans. Appl. Supercond. 25 1-12

[7] Masson P J, Rouault V R, Hoffmann G and Luongo C A 2008 Development of quench propagation models for coated conductors IEEE Trans. Appl. Supercond. 18 1321-4

[8] Badel A, Antognazza L, Decroux M and Abplanalp M 2013 Hybrid model of quench propagation in coated conductors applied to fault current limiter design IEEE Trans. Appl. Supercond. 235603705

[9] Härö E, Järvelä J and Stenvall A 2015 Variation of quench propagation velocities in YBCO cables J. Supercond. Nov. Magn. 28 1705-10

[10] Wang Y et al 2007 Detecting and describing the inhomogeneity of critical current in practical long HTS tapes using contact-free method Cryogenics 47 225-31

[11] Higashikawa K et al 2017 Characterization of critical current distribution in Roebel cable strands based on reel-to-reel scanning hall-probe microscopy IEEE Trans. Appl. Supercond. 27 1-4

[12] Hahn S, Park D K, Bascunan J and Iwasa Y 2011 HTS pancake coils without turn-to-turn insulation IEEE Trans. Appl. Supercond. 21 1592-5

[13] Lécrevisse T and Iwasa Y 2016 A (RE)BCO pancake winding with metal-as-insulation IEEE Trans. Appl. Supercond. 26 $1-5$

[14] Ciceron J, Badel A, Tixador P, Pasquet R and Forest F 2018 Test in strong background field of a modular element of a REBCO 1 MJ high energy density SMES IEEE Trans. Appl. Supercond. 28 1-5

[15] van Nugteren J et al 2018 Powering of an HTS dipole insertmagnet operated standalone in helium gas between 5 and 85 K Supercond. Sci. Technol. 31065002

[16] Noyes P D et al 2012 Protection heater development for REBCO coils IEEE Trans. Appl. Supercond. 22 4704204

[17] Levin G A, Jones W A, Novak K A and Barnes P N 2011 The effects of superconductor-stabilizer interfacial resistance on quenching of a pancake coil made out of coated conductor Supercond. Sci. Technol. 24035015
[18] Yanagisawa Y et al 2012 The mechanism of thermal runaway due to continuous local disturbances in the YBCO-coated conductor coil winding Supercond. Sci. Technol. 25075014

[19] Stenvall A, Lahtinen V and Lyly M 2014 An H-formulationbased three-dimensional hysteresis loss modelling tool in a simulation including time varying applied field and transport current: the fundamental problem and its solution Supercond. Sci. Technol. 27104004

[20] Quéval L, Zermeño V M R and Grilli F 2016 Numerical models for ac loss calculation in large-scale applications of HTS coated conductors Supercond. Sci. Technol. 29024007

[21] Chan W K and Masson P J 2010 Three-dimensional micrometer-scale modelling of quenching in high-aspectratio $\mathrm{YBa}_{2} \mathrm{Cu}_{3} \mathrm{O}_{7-\delta}$ coated conductor tapes: I. Model development and validation IEEE Trans. Appl. Supercond. 20 2370-80

[22] Cavallucci L, Breschi M, Ribani P L and Yang Y 2018 Electrothermal modeling of quench in REBCO Roebel cables IEEE Trans. Appl. Supercond. 28 1-5

[23] Breschi M, Cavallucci L, Ribani P L, Gavrilin A V and Weijers H W 2017 Modeling of quench in the coupled HTS Insert/LTS outsert magnet system of the NHMFL IEEE Trans. Appl. Supercond. 27 1-13

[24] Theva measurement devices: Tapestar ${ }^{\circledR}$. Available online: https://www.theva.com/products/\#tapestar

[25] Badel A, Escamez G and Tixador P 2015 REBCO FCL modelling: influence of local critical current nonuniformities on overall behavior for various tape architectures IEEE Trans. Appl. Supercond. 25 1-4

[26] Colangelo D and Dutoit B 2012 Inhomogeneity effects in HTS coated conductors used as resistive FCLs in medium voltage grids Supercond. Sci. Technol. 25095005

[27] Benkel T et al 2016 REBCO performance at high field with low incident angle and preliminary tests for a 10-T insert IEEE Trans. Appl. Supercond. 26 1-5

[28] Fujita S, Daibo M, Igarashi M, Kikutake R, Kakimoto K, Iijima Y, Itoh M and Saitoh T 2014 In-field critical current property of IBAD/PLD coated conductors J. Phys.: Conf. Ser. 507022007

[29] van Petegem W, Geeraerts B, Sansen W and Graindourze B 1994 Electrothermal simulation and design of integrated circuits IEEE J. Solid-State Circuits 29 143-6

[30] The MIPSE platform developed at G2Elab, Grenoble. Details available online: http://g2elab.grenoble-inp.fr/plateforms/ mipse-modeling-of-interconnected-power-systems

[31] Badel A, Tixador P, Amiet M and Brommer V 2012 SMES to supply an electromagnetic launcher IEEE Trans. Appl. Supercond. 225700204

[32] Söderlind G 2002 Automatic control and adaptive timestepping Numer. Algorithm 31 281-310

[33] Söderlind G 2003 Digital filters in adaptive time-stepping ACM Trans. Math. Softw. TOMS 29 1-26 